\title{
THE DISTRIBUTION FUNCTION IN THE MORREY SPACE
}

\author{
JOSEFINA ALVAREZ ALONSO
}

AbStRact. For $1<p<\infty$, we consider $p$-integrable functions on a finite cube $Q_{0}$ in $\mathbf{R}^{n}$, satisfying

$$
\left(\frac{1}{|Q|} \int_{Q}\left|f(x)-f_{Q}\right|^{p} d x\right)^{1 / p}<C \varphi(|Q|)
$$

for every parallel subcube $Q$ of $Q_{0}$, where $|Q|$ denotes the volume of $Q, f_{Q}$ is the mean value of $f$ over $Q$ and $\varphi(t)$ is a nonnegative function defined in $(0, \infty)$, such that $\varphi(t)$ is nonincreasing near zero, $\varphi(t) \rightarrow \infty$ as $t \rightarrow 0$, and $t \varphi^{p}(t)$ is nondecreasing near zero. The constant $C$ does not depend on $Q$. Let $g$ be a nonnegative $p$-integrable function $g:(0,1) \rightarrow \mathbf{R}$ such that $g$ is nonincreasing and $g(t) \rightarrow \infty$ as $t \rightarrow 0$. We prove here that there exist a cube $Q_{0}$ and a function $f$ satisfying condition (1) for every parallel subcube $Q$ of $Q_{0}$, such that $\delta_{f}(\lambda)>C_{1} \delta_{g}(\lambda)$ for $\lambda>\lambda_{0}, C_{1}>0$, where $\delta(\lambda)$ denotes the distribution function.

John and Nirenberg have introduced in [1] the functions of bounded mean oscillation. An integrable function $f$ on a finite cube $Q_{0}$ in $\mathbf{R}^{n}$ is called a BMO function if there exists a constant $C>0$ such that

$$
\frac{1}{|Q|} \int_{Q}\left|f(x)-f_{Q}\right| d x \leqslant C
$$

for every parallel subcube $Q$ of $Q_{0}$, where $|Q|$ denotes the volume of $Q$ and $f_{Q}$ is the mean value of $f$ over $Q$.

These authors have shown that the distribution function of a function of bounded mean oscillation decreases exponentially. More exactly, there exist constants $C, \alpha>0$ such that

$$
\delta_{f-f_{Q_{0}}}(\lambda)=\operatorname{meas}\left\{x \in Q_{0}|| f(x)-f_{Q_{0}} \mid>\lambda\right\} \leqslant C e^{-\alpha \lambda}\left|Q_{0}\right| \quad \text { for every } \lambda>0 .
$$

This implies that the BMO functions satisfy additional conditions. Actually, they belong to $L^{p}\left(Q_{0}\right)$ for all $p<\infty$ and they satisfy

$$
\left(\frac{1}{|Q|} \int_{Q}\left|f(x)-f_{Q}\right|^{p} d x\right)^{1 / p} \leqslant C .
$$

Presented at the meeting of the Union Matemática Argentina, August 1980; received by the editors October 20, 1980.

AMS (MOS) subject classifications (1970). Primary 43A15, 46E30; Secondary 26A09.

Key words and phrases. Mean oscillation, distribution function, Morrey space. 
Now, we are led to consider more general spaces, for instance, those $p$-integrable functions $f$ on $Q_{0}$ satisfying

$$
\left(\frac{1}{|Q|} \int_{Q}\left|f(x)-f_{Q}\right|^{p} d x\right)^{1 / p} \leqslant C|Q|^{-\alpha} \text { for some } 0<\alpha<1 / p .
$$

It would be interesting to obtain some estimate for the distribution function in this space. However, it does not seem to be possible. Actually, we show this in a more general space.

In fact, let $\varphi(t)$ be a nonnegative function defined in $(0, \infty)$ such that $\varphi(t)$ does not increase near zero, $\varphi(t) \rightarrow \infty$ as $t \rightarrow 0$, and $t \varphi^{p}(t)$ does not decrease near zero. We say that a function $f$ that is $p$-integrable over a cube $Q_{0}$ in $\mathbf{R}^{n}$ belongs to the space $M_{\varphi}^{p}\left(Q_{0}\right)$ if it satisfies $\left(|Q|^{-1} \int_{Q}\left|f(x)-f_{Q}\right|^{p} d x\right)^{1 / p}<C \varphi(|Q|)$ for every parallel subcube $Q$ of $Q_{0}$.

When $\varphi(t)=t^{-\alpha}, 0<\alpha<1 / p$, we get the Morrey space, that is the functions satisfying (2). Now, we prove the following result.

Proposition. Let $g:(0,1) \rightarrow \mathbf{R}$ be a nonnegative, nonincreasing p-integrable function such that $g(t) \rightarrow \infty$ as $t \rightarrow 0$. Then, there exist a cube $Q_{0}$, a function $f \in M_{\varphi}^{p}\left(Q_{0}\right)$ and two constants $C_{1}, \lambda_{0}>0$ such that

$$
\delta_{f}(\lambda) \geqslant C_{1} \delta_{g}(\lambda) \text { for } \lambda \geqslant \lambda_{0} \text {. }
$$

Proof. First, we prove the assertion in one variable. The general case will follow from this one.

According to the hypothesis, there exists $a \leqslant 1$ such that $\varphi(t)$ does not increase for $t \leqslant a, t \varphi^{p}(t)$ does not decrease there, and $\varphi(a)>0$. We can also suppose that $\varphi(a)=1$, so we get $\varphi(t) \geqslant 1$ for $t \leqslant a$. We suppose also that $\int_{0}^{1} g(t)^{p} d t<a / 4^{p}$. On the other hand, we complete the definition of the function $g$ in such a way that it remains left continuous.

Let $h \geqslant 2$ be the first natural number such that $g(t)<2^{h-1}$, for some $t$. For each $k \geqslant h+1$, we consider the interval $I_{k}=\left(x_{k}, x_{k-1}\right)$, such that $2^{k-2}<g(t)<2^{k-1}$ in $I_{k}$.

We assert that the sequence $\left\{x_{k}\right\}$ converges to zero. In fact, since it is decreasing, it has a limit $L \geqslant 0$. $L$ must be zero because, by construction, we have $g\left(x_{k}\right) \geqslant$ $2^{k-2}, k \geqslant h+1$. Furthermore, the length of the intervals $I_{k}$ decreases as a geometric progression. In fact,

$$
\left|I_{k}\right|^{p(k-2)} \leqslant \int_{I_{k}} g(t)^{p} d t \leqslant \frac{a}{4^{p}}
$$

so that $\left|I_{k}\right| \leqslant a / 2^{p k}$ for $k \geqslant h+1$.

Now, we define a step function $m(t)$ as

$$
m(t)=2^{k-1} \text { in } I_{k}, \text { for } k \geqslant h+1 .
$$

Clearly, $\delta_{m}(\lambda) \geqslant \delta_{g}(\lambda)$ for $\lambda \geqslant 2^{h-1}$. Now, we will replace each interval $I_{k}$ by another one, $J_{k}=\left(y_{k}, y_{k-1}\right)$, of length $\left|J_{k}\right|=2^{p k}\left|I_{k}\right|=2^{p} \int_{I_{k}} m(t)^{p} d t$. 


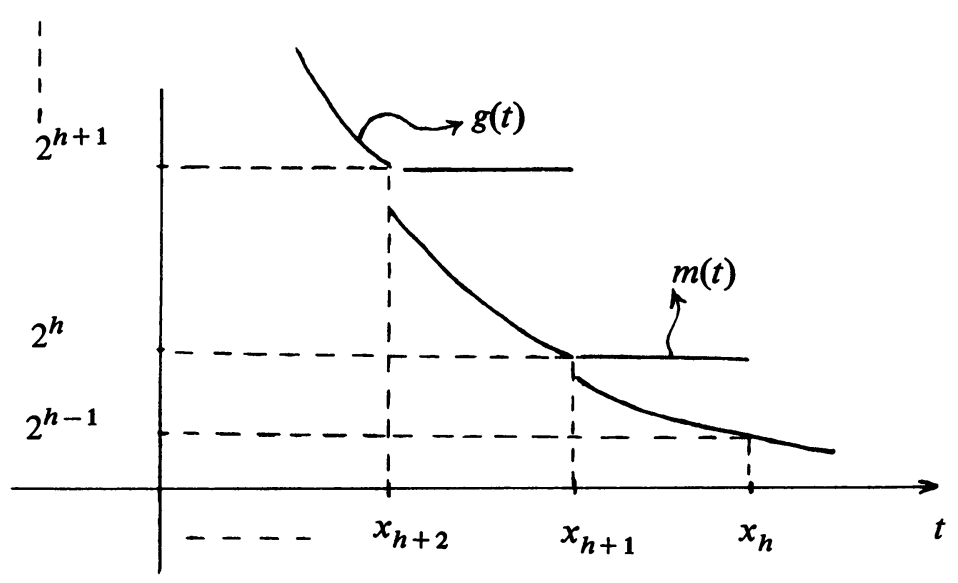

We assert that $\Sigma_{k>h+1}\left|J_{k}\right|<\infty$. In fact,

$$
\begin{aligned}
\sum_{k>h+1}\left|J_{k}\right| & =\sum_{k>h+1} 2^{p k}\left|I_{k}\right|=4^{p} \sum_{k>h+1} 2^{p(k-2)}\left|I_{k}\right| \\
& \leqslant 4^{p} \sum_{k>h+1} \int_{I_{k}} g(t)^{p} d t \leqslant 4^{p} \int_{0}^{1} g(t)^{p} d t<a .
\end{aligned}
$$

Now, we will define a function $f(t)$ on the interval $\left(0, y_{h}\right)$ in the following way. We fix one interval $I_{k}$ and we divide it into $n_{k}$ subintervals of length $\delta_{k}=\left|I_{k}\right| / n_{k}$. The number $n_{k}$ will be selected later. Now, let $\varepsilon_{k}=\left(\left|J_{k}\right|-\left|I_{k}\right|\right) /\left(n_{k}-1\right)$. We divide the interval $J_{k}$ into $2 n_{k}-1$ subintervals of length $\delta_{k}$ and $\varepsilon_{k}$ alternatively. We define $f(t)$ as $2^{k-1}$ in the intervals of length $\delta_{k}$ and zero in the others.

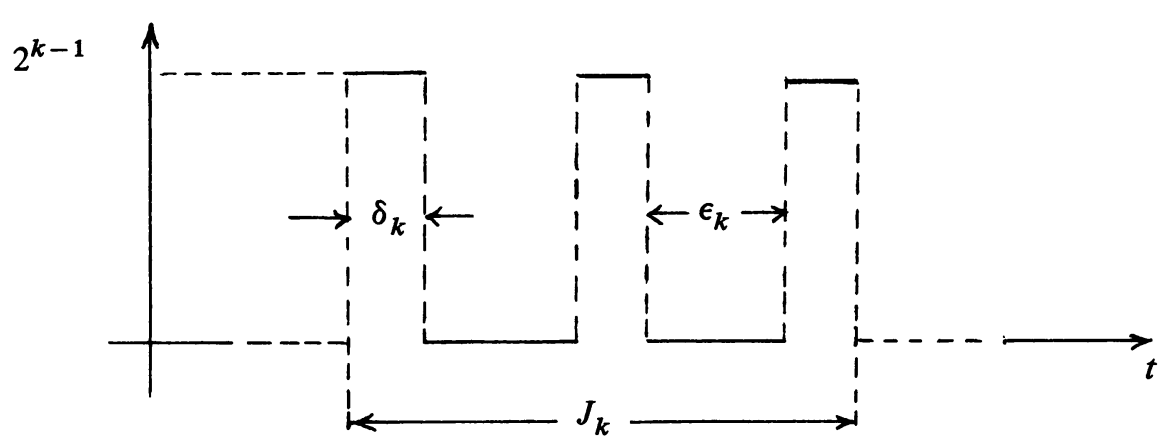

Over each interval $J_{k}$ the measure of the set where $f(t)$ does not vanish is exactly $\left|I_{k}\right|$. Furthermore, $f(t)$ coincides with $m(t)$ on that set. So, both functions have the same distribution function.

Now, we assert that selecting the number $n_{k}$ in each interval $J_{k}$ in a correct way, we get $f(t) \in M_{\varphi}^{P}\left(\left(0, y_{h}\right)\right)$. Actually, we will prove that there exists a constant $C>0$ such that

$$
\int_{J} f(t)^{p} d t \leqslant C|J| \varphi^{p}(|J|)
$$

for every subinterval $J$ of $\left(0, y_{h}\right)$. This will clearly imply that $f \in M_{\varphi}^{P}\left(\left(0, y_{h}\right)\right)$. 
First, let us consider the interval $J=\left(0, y_{L-1}\right)$, for some $L$,

$$
\begin{aligned}
\int_{J} f(t)^{p} d t & =\sum_{k>L} \int_{J_{k}} f(t)^{p} d t=\sum_{k>L} 2^{p(k-1)}\left|I_{k}\right| \\
& =2^{-p} \sum_{k>L}\left|J_{k}\right|=2^{-p}|J| .
\end{aligned}
$$

As was shown above, $\Sigma_{k>L}\left|J_{k}\right| \leqslant a$; thus, $\varphi(|J|) \geqslant 1$ or $|J| \varphi^{p}(|J|) \geqslant|J|$. So, we get

$$
\int_{J} f(t)^{p} d t \leqslant 2^{-p}|J| \varphi^{p}(|J|)
$$

Now, let us consider an interval $J=\left(y_{s}, y_{L-1}\right)$. In the same way, we obtain

$$
\begin{aligned}
\int_{J} f(t)^{p} d t & =\sum_{L}^{s} \int_{J_{k}} f(t)^{p} d t=\sum_{L}^{s} 2^{p(k-1)}\left|I_{k}\right| \\
& =2^{-p} \sum_{L}^{s}\left|J_{k}\right|=2^{-p}|J| \leqslant 2^{-p}|J| \varphi^{p}(|J|) .
\end{aligned}
$$

Now, we will consider an interval $J$ contained in one of the intervals $J_{k}$. We will select the number $n_{k}$ in order to obtain the desired inequality over this interval.

Since we have supposed the index $k$ to be fixed, we will write simply $n, \delta, \varepsilon$. We first assume that there are $j$ intervals of length $\delta$, which cover the interval $J$, in the following sense.

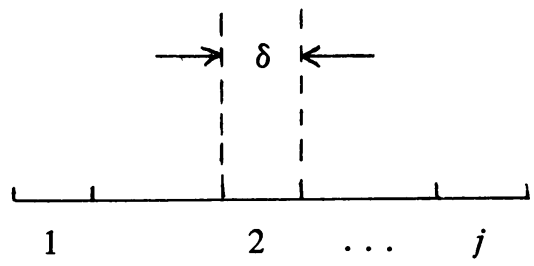

It would be desirable to obtain the inequality

$$
2^{p(k-1)} j \delta \leqslant(j \delta+(j-1) \varepsilon) \varphi^{p}(j \delta+(j-1) \varepsilon), \quad 1 \leqslant j \leqslant n .
$$

Since $\delta=\left|I_{k}\right| / n, \varepsilon=\left(\left|J_{k}\right|-\left|I_{k}\right|\right) / n-1=\left(2^{p k}-1\right)\left|I_{k}\right| / n-1$, we can write the above inequality in the form

$$
\begin{aligned}
& 2^{p(k-1)} j \frac{\left|I_{k}\right|}{n} \\
& \quad \leqslant\left(j \frac{\left|I_{k}\right|}{n}+(j-1)\left(2^{p k}-1\right) \frac{\left|I_{k}\right|}{n-1}\right) \varphi^{p}\left(j \frac{\left|I_{k}\right|}{n}+(j-1)\left(2^{p k}-1\right) \frac{\left|I_{k}\right|}{n-1}\right)
\end{aligned}
$$

or,

$$
2^{p(k-1)} \leqslant\left(1+\frac{n}{j} \frac{j-1}{n-1}\left(2^{p k}-1\right)\right) \varphi^{p}\left(\left|I_{k}\right|\left(\frac{j}{n}+\frac{j-1}{n-1}\left(2^{p k}-1\right)\right)\right) .
$$

As we saw above, $\left|I_{k}\right| \leqslant a / 2^{p k}$. Moreover, $(j-1) /(n-1) \leqslant j / n$. Thus,

$$
\left|I_{k}\right|\left(\frac{j}{n}+\frac{j-1}{n-1}\left(2^{p k}-1\right)\right) \leqslant \frac{a}{2^{p k}}\left(\frac{j}{n}+\frac{j}{n}\left(2^{p k}-1\right)\right)=a \frac{j}{n} \leqslant a .
$$


Since we have supposed that $\varphi$ is a nonincreasing function for $t<a$, we obtain

$$
\varphi\left(\left|I_{k}\right|\left(\frac{j}{n}+\frac{j-1}{n-1}\left(2^{p k}-1\right)\right)\right)>\varphi\left(a \frac{j}{n}\right)>\varphi(a)=1 .
$$

Thus, it suffices to find a natural number $n$ so that

$$
2^{p(k-1)} \leqslant\left(1+\frac{n}{j} \frac{j-1}{n-1}\left(2^{p k}-1\right)\right) \varphi^{p}\left(a \frac{j}{n}\right), \quad 1<j<n,
$$

for $k \geqslant h+1$ fixed. Since $\varphi(t) \rightarrow \infty$ as $t \rightarrow 0$, there exists $0<r(k, p)<a$ such that $a j / n \leqslant r$ which implies $2^{p(k-1)} \leqslant \varphi^{p}(a j / n)$. Thus, when $j / n<r / a$, we get the desired inequality.

Now, we suppose $1 \leqslant n / j \leqslant a / r$, and we will select $n$ in such a way that

$$
2^{p(k-1)} \leqslant 1+\frac{n}{j} \frac{j-1}{n-1}\left(2^{p k}-1\right) .
$$

Since $(j-1) / j=1-1 / j$ increases as $j$ increases, the worst case occurs when $n / j=a / r$; that is,

$$
2^{p(k-1)} \leqslant 1+\frac{a}{r} \frac{n r / a-1}{n-1}\left(2^{p k}-1\right) .
$$

From this inequality, we deduce that selecting $n \geqslant(a / r-\theta) /(1-\theta)$, where $\theta=\left(2^{p(k-1)}-1\right) /\left(2^{p k}-1\right)$, we obtain the desired inequality for the subinterval $J$. Now, we suppose that $J$ is contained in one of the intervals of length $\delta_{k}$, for $k$ fixed.

In this case, it suffices to satisfy the inequality

$$
2^{p(k-1)}|J| \leqslant|J| \varphi^{p}(|J|),
$$

or, $2^{k-1} \leqslant \varphi(|J|)$.

Since $|J| \leqslant \delta_{k}$, we will have $\varphi(|J|) \geqslant \varphi\left(\delta_{k}\right)$; so that, it suffices to obtain $2^{k-1} \leqslant$ $\varphi\left(\delta_{k}\right)$. But this is the inequality above, for $j=1$.

In the same way, we can prove the inequality for a given subinterval $J$ of some interval $J_{k}$. We merely have to use that the function $t \varphi^{p}(t)$ does not decrease for $t \leqslant a$.

Finally, let us consider a subinterval $J$ of the interval $\left(0, y_{h}\right)$. We can divide $J$ into at most three intervals. One of them is a union of some intervals $J_{k}$, and the others are contained in some other intervals $J_{k^{\prime}}$ and $J_{k^{\prime \prime}}$. Thus, according to all we have said above, and using again the fact that $t \varphi^{p}(t)$ is a nondecreasing function, we obtain the inequality. This concludes the one variable case.

In the general case, we argue as follows. Let $f(t)$ be a function in the space $M_{\varphi}^{p}\left(\left(0, y_{h}\right)\right)$, satisfying the desired hypothesis. Let $Q_{0}=\left\{\left(t_{1}, \ldots, t_{n}\right) \mid 0<t_{j}<y_{h}\right.$, $j=1, \ldots, n\}$. We define the function $F\left(t_{1}, \ldots, t_{n}\right)$ as

$$
F\left(t_{1}, \ldots, t_{n}\right)=f\left(t_{1}\right)
$$

We assert that $F \in M_{\varphi}^{p}\left(Q_{0}\right)$.

In fact, let $Q$ be a parallel subcube of $Q_{0}$; we can write $Q=S_{1} \times \cdots \times S_{n}$, where $S_{j}$ are subintervals of the same length of $\left(0, y_{h}\right)$. Thus,

$$
\int_{Q} F\left(t_{1}, \ldots, t_{n}\right)^{p} d t_{1} \cdots d t_{n}=\left|S_{2}\right| \cdots\left|S_{n}\right| \int_{S_{1}} f\left(t_{1}\right)^{p} d t_{1}<C|Q| \varphi^{p}\left(\left|S_{1}\right|\right) .
$$


Since $\left|S_{j}\right|<y_{h} \leqslant a \leqslant 1$, we get $|Q|=\left|S_{1}\right| \cdots\left|S_{n}\right|<\left|S_{1}\right|<a$. So that

$$
\varphi\left(\left|S_{1}\right|\right)<\varphi(|Q|) \text { or } \varphi^{p}\left(\left|S_{1}\right|\right)<\varphi^{p}(|Q|) .
$$

On the other hand, we have also that $\delta_{F}(\lambda)=y_{h}^{n-1} \delta_{f}(\lambda)$ for $\lambda>0$. This completes the proof.

REMARK. In [2], the definition of the Morrey space appears in a slightly different way. Working over cubes, that definition may be stated as follows.

A $p$-integrable function $f$ on a finite cube $Q_{0}$ in $\mathbf{R}^{n}$ belongs to the Morrey space of order $\alpha, 0<\alpha<1 / p$, if

$$
\sup _{\substack{x \in \bar{Q}_{0} \\|Q(x)|<\left|Q_{0}\right|}} \inf _{c \in \mathbf{C}}\left[|Q(x)|^{p \alpha-1} \int_{Q(x) \cap Q_{0}}|f(y)-c|^{p} d y\right]^{1 / p}<\infty
$$

where $\bar{Q}_{0}$ means the closure of $Q_{0}$ and, given $x \in \bar{Q}_{0}, Q(x)$ is a cube centered in $x$, parallel to $Q_{0}$.

Actually, Campanato has shown that it is the same to consider

$$
\sup _{\substack{x \in \bar{Q}_{0} \\|Q(x)|<\left|Q_{0}\right|}}\left[|Q(x)|^{p \alpha-1} \int_{Q(x) \cap Q_{0}}|f(y)|^{p} d y\right]^{1 / p}<\infty
$$

(see [3]). From this, we are led to consider those $p$-integrable functions on $Q_{0}$ such that

$$
\int_{Q(x) \cap Q_{0}}|f(y)|^{p} d y \leqslant C|Q(x)| \varphi^{p}(|Q(x)|) \quad \text { for all } x \in \bar{Q}_{0},|Q(x)| \leqslant\left|Q_{0}\right| .
$$

The function $F\left(t_{1}, \ldots, t_{n}\right)$ constructed in the above proposition satisfies this inequality, in fact let $Q(x) \cap Q_{0}=S_{1} \times \cdots \times S_{n}$, where $S_{j}$ are subintervals of $\left(0, y_{h}\right)$. Then

$$
\begin{aligned}
\int_{Q(x) \cap Q_{0}} F\left(t_{1}, \ldots, t_{n}\right)^{p} d t_{1} \cdots d t_{n} & =\left|S_{2}\right| \cdots\left|S_{n}\right| \int_{S_{1}} f\left(t_{1}\right)^{p} d t_{1} \\
& \leqslant C\left|Q(x) \cap Q_{0}\right| \varphi^{p}\left(\left|S_{1}\right|\right) .
\end{aligned}
$$

Since $\left|S_{j}\right| \leqslant y_{h} \leqslant a \leqslant 1$, we have $a \geqslant\left|S_{1}\right| \geqslant\left|S_{1}\right| \cdots\left|S_{n}\right|=\left|Q(x) \cap Q_{0}\right|$. The function $\varphi(t)$ is nonincreasing and the function $t \varphi^{p}(t)$ is nondecreasing for $t<a$, so we get

$$
\left|Q(x) \cap Q_{0}\right| \varphi^{p}\left(\left|S_{1}\right|\right) \leqslant\left|Q(x) \cap Q_{0}\right| \varphi^{p}\left(\left|Q(x) \cap Q_{0}\right|\right) \leqslant|Q(x)| \varphi^{p}(|Q(x)|) .
$$

Actually, we have proved that

$$
\int_{Q(x) \cap Q_{0}} F\left(t_{1}, \ldots, t_{n}\right)^{p} d t_{1} \cdots d t_{n}<C\left|Q(x) \cap Q_{0}\right| \varphi^{p}\left(\left|Q(x) \cap Q_{0}\right|\right) .
$$

This concludes the remark. 
Acknowledgement. We thank Professor A. P. Calderón for his very helpful suggestions.

\section{REFERENCES}

1. F. John and L. Nirenberg, On functions of bounded mean ascillation, Comm. Pure Appl. Math. 14 (1961), 415-426.

2. J. Peetre, On the theory of $L_{p, \lambda}$ spaces, J. Funct. Anal. 4 (1969), 71-87.

3. S. Campanato, Proprietà di una famiglia di spazi funzionali, Ann. Scuola Norm. Sup. Pisa Cl. Sci. (4) 18 (1964), 137-160.

facultad de Cienclas Exactas, Universidad de Buenos Arres, Consejo Nacional de InvestigaCiones, Buenos Aires, Argentina 